\title{
The average value inequality in sequential effect
}

\author{
algebras*
}

\author{
Shen Jun ${ }^{1,2}$, Wu Junde ${ }^{1 \dagger}$ \\ ${ }^{1}$ Department of Mathematics, Zhejiang University, Hangzhou 310027, P. R. China \\ ${ }^{2}$ Department of Mathematics, Anhui Normal University, Wuhu 241003, P. R. China
}

\begin{abstract}
A sequential effect algebra $(E, 0,1, \oplus, \circ)$ is an effect algebra on which a sequential product $\circ$ with certain physics properties is defined, in particular, sequential effect algebra is an important model for studying quantum measurement theory. In 2005, Gudder asked the following problem: If $a, b \in(E, 0,1, \oplus, \circ)$ and $a \perp b$ and $a \circ b \perp a \circ b$, is it the case that $2(a \circ b) \leq a^{2} \oplus b^{2}$

? In this paper, we construct an example to answer the problem negatively.
\end{abstract}

Key Words. Effect algebras, Sequential effect algebras, Average value inequality.

MR(2000) Subject Classification. 81P15

Effect algebra was introduced in 1994 to model the quantum logic which may be fuzzy or unsharp, to be precise, an effect algebra is a system $(E, 0,1, \oplus)$, where 0 and 1 are distinct elements of $E$ and $\oplus$ is a partial binary operation on $E$ satisfying [1]:

(EA1) If $a \oplus b$ is defined, then $b \oplus a$ is defined and $b \oplus a=a \oplus b$.

(EA2) If $a \oplus(b \oplus c)$ is defined, then $(a \oplus b) \oplus c$ is defined and

$$
(a \oplus b) \oplus c=a \oplus(b \oplus c)
$$

\footnotetext{
*This project is supported by Natural Science Found of China (10771191 and 10471124).

${ }^{\dagger}$ E-mail: wjd@zju.edu.cn
} 
(EA3) For each $a \in E$, there exists a unique element $b \in E$ such that $a \oplus b=1$.

(EA4) If $a \oplus 1$ is defined, then $a=0$.

In an effect algebra $(E, 0,1, \oplus)$, if $a \oplus b$ is defined, we write $a \perp b$. If $a \perp a$, we denote $a \oplus a$ by $2 a$. For each $a \in(E, 0,1, \oplus)$, it follows from (EA3) that there exists a unique element $b \in E$ such that $a \oplus b=1$, we denote $b$ by $a^{\prime}$. If $a \wedge a^{\prime}=0$, we say that $a$ is a sharp element of $(E, 0,1, \oplus)$ (see $[2])$. Let $a, b \in(E, 0,1, \oplus)$, if there exists a $c \in E$ such that $a \perp c$ and $a \oplus c=b$, then we say that $a \leq b$. It follows from [1] that $\leq$ is a partial order of $(E, 0,1, \oplus)$ and satisfies that for each $a \in E$, $0 \leq a \leq 1, a \perp b$ iff $a \leq b^{\prime}$.

In 2001, in order to study quantum measurement theory, Gudder began to consider the sequential product of two measurements $A$ and $B$ (see [3]). In 2002, Professors Gudder and Greechie introduced the abstract sequential effect algebra structure, that is:

A sequential effect algebra is an effect algebra $(E, 0,1, \oplus)$ and another binary operation o defined on $(E, 0,1, \oplus)$ satisfying [4]:

(SEA1) The map $b \mapsto a \circ b$ is additive for each $a \in E$, that is, if $b \perp c$, then $a \circ b \perp a \circ c$ and $a \circ(b \oplus c)=a \circ b \oplus a \circ c$.

(SEA2) $1 \circ a=a$ for each $a \in E$.

(SEA3) If $a \circ b=0$, then $a \circ b=b \circ a$.

(SEA4) If $a \circ b=b \circ a$, then $a \circ b^{\prime}=b^{\prime} \circ a$ and $a \circ(b \circ c)=(a \circ b) \circ c$ for each $c \in E$.

(SEA5) If $c \circ a=a \circ c$ and $c \circ b=b \circ c$, then $c \circ(a \circ b)=(a \circ b) \circ c$ and $c \circ(a \oplus b)=(a \oplus b) \circ c$ whenever $a \perp b$.

Let $(E, 0,1, \oplus, \circ)$ be a sequential effect algebra. Then the operation $\circ$ is said to be a sequential product on $(E, 0,1, \oplus, \circ)$. If $a, b \in(E, 0,1, \oplus, \circ)$ and $a \circ b=b \circ a$, then we say that $a$ and $b$ is sequentially independent and denoted by $a \mid b$ (see [4]). If $a \in(E, 0,1, \oplus, \circ)$, we denote $a \circ a$ by $a^{2}$, it follows from ([4, Lemma 3.2]) that $a$ is a sharp element of $(E, 0,1, \oplus, \circ)$ iff $a^{2}=a$. We denote the set of all sharp elements in $(E, 0,1, \oplus, \circ)$ by $E_{s}$. 
In 2005, in order to motivate the study of sequential effect algebra theory, Professor Gudder presented 25 important and interesting problems, the 23th problem asked ([5]): If $a, b \in(E, 0,1, \oplus, \circ)$ and $a \perp b$ and $a \circ b \perp a \circ b$, is it the case that $2(a \circ b) \leq a^{2} \oplus b^{2}$ ? In this paper, we construct an example to answer the problem negatively.

At first, we show that the above average value inequality does hold in the underlying sequential effect algebras under some additional conditions. That is:

Proposition 1. If $(E, 0,1, \oplus, \circ)$ is a sequential effect algebra, $a, b \in E, a^{2} \perp b^{2}$ (a sufficient condition for this is $a \perp b), a \leq b$ (or $b \leq a)$ and $a \mid b$, then $(a \circ b) \perp(a \circ b)$ and $2(a \circ b) \leq a^{2} \oplus b^{2}$.

Proof. Since $a \leq b$, there exists a $c \in E$ such that $a \oplus c=b$. Since $a \mid b$, it follows that $c \mid b$ (see [4] Lemma 3.1(v)).

$$
\begin{aligned}
& c \circ b=c \circ(a \oplus c)=(c \circ a) \oplus c^{2} . \\
& b^{2}=b \circ(a \oplus c)=(b \circ a) \oplus(b \circ c)=(a \circ b) \oplus(c \circ b)=(a \circ b) \oplus(c \circ a) \oplus c^{2} .
\end{aligned}
$$

Since $a^{2} \perp b^{2}, a^{2} \oplus b^{2}=a^{2} \oplus(a \circ b) \oplus(c \circ a) \oplus c^{2}$.

While $a \circ b=a \circ(a \oplus c)=a^{2} \oplus(a \circ c)=a^{2} \oplus(c \circ a)$, so $a^{2} \oplus b^{2}=(a \circ b) \oplus(a \circ b) \oplus c^{2}$.

It follows that $(a \circ b) \perp(a \circ b)$ and $2(a \circ b) \leq a^{2} \oplus b^{2}$.

Finally, if $a \perp b$, it follows from $a^{2} \leq a$ and $b^{2} \leq b$ that $a^{2} \perp b^{2}$. The proposition is proved.

Proposition 2. If $(E, 0,1, \oplus, \circ)$ is a sequential effect algebra, $a, b \in E, a \perp b$, $a \in E_{s}\left(\right.$ or $\left.b \in E_{s}\right)$, then $(a \circ b) \perp(a \circ b)$ and $2(a \circ b) \leq a^{2} \oplus b^{2}$.

Proof. Since $a \perp b$ and $a \in E_{s}$, it follows that $a \circ b=0$ (see [4] Lemma 3.3(ii)), so $2(a \circ b) \leq a^{2} \oplus b^{2}$.

Now, we construct a sequential effect algebra to show that the above average value inequality does not always hold.

In this paper, we denote $\mathbf{Z}$ the integer set, $\mathbf{N}$ the nonnegative integer set and $\mathbf{N}^{+}$the positive integer set.

Let $E_{0}=\left\{0,1, a_{n}, b_{n}, c_{i, k, m}, d_{i, k, m} \mid n \in \mathbf{N}^{+}, i, k \in \mathbf{N}\right.$ and $\left.i^{2}+k^{2} \neq 0, m \in \mathbf{Z}\right\}$. 
For simplicity, in the sequel, unless specified, the subindex of respective elements will always take values in the corresponding default sets. To be accurately, when we write $a_{n}, b_{n}, n$ always take values in $\mathbf{N}^{+}$, when we write $c_{i, k, m}, d_{i, k, m}, i, k$ always take values in $\mathbf{N}$ and $i^{2}+k^{2} \neq 0$ and $m$ always take values in $\mathbf{Z}$.

We define a partial binary operation $\oplus$ on $E_{0}$ as follows(when we write $x \oplus y=z$, we always mean $x \oplus y=z=y \oplus x)$ :

For each $x \in E_{0}, 0 \oplus x=x$,

$a_{n} \oplus a_{m}=a_{n+m}, a_{n} \oplus c_{i, k, m}=c_{i, k, n+m}, a_{n} \oplus d_{i, k, m}=d_{i, k, m-n}, c_{i, k, m} \oplus c_{r, s, t}=$ $c_{i+r, k+s, m+t}$.

For $n<m, a_{n} \oplus b_{m}=b_{m-n}, a_{n} \oplus b_{n}=1$.

For $i \leq r$ and $k \leq s$ and $(r-i)^{2}+(s-k)^{2} \neq 0 . c_{i, k, m} \oplus d_{r, s, t}=d_{r-i, s-k, t-m}$.

For $i=r$ and $k=s$ and $m<t, c_{i, k, m} \oplus d_{r, s, t}=b_{t-m}$.

For $i=r$ and $k=s$ and $m=t, c_{i, k, m} \oplus d_{r, s, t}=1$.

No other $\oplus$ operation is defined.

Next, we define a binary operation $\circ$ on $E_{0}$ as follows(when we write $x \circ y=z$, we always mean $x \circ y=z=y \circ x)$ :

For each $x \in E_{0}, 0 \circ x=0,1 \circ x=x$,

$a_{n} \circ a_{m}=0, a_{n} \circ b_{m}=a_{n}, b_{n} \circ b_{m}=b_{m+n}, a_{n} \circ c_{i, k, m}=0, c_{i, k, m} \circ b_{n}=c_{i, k, m}$, $a_{n} \circ d_{i, k, m}=a_{n}, b_{n} \circ d_{i, k, m}=d_{i, k, m+n}, d_{i, k, m} \circ d_{r, s, t}=d_{i+r, k+s, m+t-i s-k r}, c_{i, k, m} \circ d_{r, s, t}=$ $c_{i, k, m-i s-k r}, c_{i, k, m} \circ c_{r, s, t}=a_{i s+k r}($ when $i s+k r \neq 0)$ or $0($ when is $+k r=0)$.

Proposition 3. $\left(E_{0}, 0,1, \oplus, \circ\right)$ is a sequential effect algebra.

Proof. First we verify that $\left(E_{0}, 0,1, \oplus\right)$ is an effect algebra.

(EA1) and (EA4) are trivial.

We verify (EA2), we omit the trivial cases about 0,1 :

$a_{n} \oplus\left(a_{m} \oplus a_{k}\right)=\left(a_{n} \oplus a_{m}\right) \oplus a_{k}=a_{k+m+n}$.

$a_{n} \oplus\left(a_{m} \oplus c_{i, j, k}\right)=\left(a_{n} \oplus a_{m}\right) \oplus c_{i, j, k}=c_{i, j, k+m+n}$.

$a_{n} \oplus\left(a_{m} \oplus d_{i, j, k}\right)=\left(a_{n} \oplus a_{m}\right) \oplus d_{i, j, k}=d_{i, j, k-m-n}$.

$a_{n} \oplus\left(c_{r, s, t} \oplus c_{i, j, k}\right)=\left(a_{n} \oplus c_{r, s, t}\right) \oplus c_{i, j, k}=c_{i+r, s+j, k+t+n}$.

$c_{l, m, n} \oplus\left(c_{r, s, t} \oplus c_{i, j, k}\right)=\left(c_{l, m, n} \oplus c_{i, j, k}\right) \oplus c_{r, s, t}=c_{i+l+r, j+m+s, k+n+t}$.

Each $a_{n} \oplus\left(a_{m} \oplus b_{k}\right)$ or $\left(a_{n} \oplus a_{m}\right) \oplus b_{k}$ is defined iff $n+m \leq k$, at this case, 
$a_{n} \oplus\left(a_{m} \oplus b_{k}\right)=\left(a_{n} \oplus a_{m}\right) \oplus b_{k}=b_{k-m-n}($ when $m+n<k)$ or $1($ when $m+n=k)$.

Each $a_{n} \oplus\left(c_{r, s, t} \oplus d_{i, j, k}\right)$ or $\left(a_{n} \oplus c_{r, s, t}\right) \oplus d_{i, j, k}$ or $\left(a_{n} \oplus d_{i, j, k}\right) \oplus c_{r, s, t}$ is defined iff one of the following two conditions is satisfied:

(1) $r \leq i$ and $s \leq j$ and $(i-r)^{2}+(j-s)^{2} \neq 0$, at this case, $a_{n} \oplus\left(c_{r, s, t} \oplus d_{i, j, k}\right)=$ $\left(a_{n} \oplus c_{r, s, t}\right) \oplus d_{i, j, k}=\left(a_{n} \oplus d_{i, j, k}\right) \oplus c_{r, s, t}=d_{i-r, j-s, k-t-n} ;$

(2) $r=i$ and $s=j$ and $n+t \leq k$, at this case, $a_{n} \oplus\left(c_{r, s, t} \oplus d_{i, j, k}\right)=\left(a_{n} \oplus c_{r, s, t}\right) \oplus$ $d_{i, j, k}=\left(a_{n} \oplus d_{i, j, k}\right) \oplus c_{r, s, t}=b_{k-t-n}($ when $n+t<k)$ or $1($ when $n+t=k)$.

Each $c_{l, m, n} \oplus\left(c_{r, s, t} \oplus d_{i, j, k}\right)$ or $\left(c_{l, m, n} \oplus c_{r, s, t}\right) \oplus d_{i, j, k}$ is defined iff one of the following two conditions is satisfied:

(1) $l+r \leq i$ and $m+s \leq j$ and $(i-l-r)^{2}+(j-m-s)^{2} \neq 0$, at this case, $c_{l, m, n} \oplus\left(c_{r, s, t} \oplus d_{i, j, k}\right)=\left(c_{l, m, n} \oplus c_{r, s, t}\right) \oplus d_{i, j, k}=d_{i-l-r, j-m-s, k-t-n} ;$

(2) $l+r=i$ and $m+s=j$ and $n+t \leq k$, at this case, $c_{l, m, n} \oplus\left(c_{r, s, t} \oplus d_{i, j, k}\right)=$ $\left(c_{l, m, n} \oplus c_{r, s, t}\right) \oplus d_{i, j, k}=b_{k-t-n}($ when $n+t<k)$ or $1($ when $n+t=k)$.

We verify (EA3): $a_{n} \oplus b_{n}=1, c_{i, k, m} \oplus d_{i, k, m}=1$.

So $\left(E_{0}, 0,1, \oplus\right)$ is an effect algebra.

We now verify that $\left(E_{0}, 0,1, \oplus, \circ\right)$ is a sequential effect algebra.

(SEA2) and (SEA3) and (SEA5) are trivial.

We verify (SEA1), we omit the trivial cases about 0,1 :

$$
\begin{aligned}
& a_{n} \circ\left(a_{m} \oplus a_{k}\right)=a_{n} \circ a_{m} \oplus a_{n} \circ a_{k}=0, \\
& b_{n} \circ\left(a_{m} \oplus a_{k}\right)=b_{n} \circ a_{m} \oplus b_{n} \circ a_{k}=a_{m+k}, \\
& c_{r, s, t} \circ\left(a_{m} \oplus a_{k}\right)=c_{r, s, t} \circ a_{m} \oplus c_{r, s, t} \circ a_{k}=0, \\
& d_{r, s, t} \circ\left(a_{m} \oplus a_{k}\right)=d_{r, s, t} \circ a_{m} \oplus d_{r, s, t} \circ a_{k}=a_{m+k} . \\
& a_{n} \circ\left(a_{m} \oplus c_{r, s, t}\right)=a_{n} \circ a_{m} \oplus a_{n} \circ c_{r, s, t}=0, \\
& b_{n} \circ\left(a_{m} \oplus c_{r, s, t}\right)=b_{n} \circ a_{m} \oplus b_{n} \circ c_{r, s, t}=c_{r, s, m+t}, \\
& c_{x, y, z} \circ\left(a_{m} \oplus c_{r, s, t}\right)=c_{x, y, z} \circ a_{m} \oplus c_{x, y, z} \circ c_{r, s, t}=a_{x s+y r} \text { when xs+yr } \neq
\end{aligned}
$$

$0)$ or $0($ when $x s+y r=0)$,

$$
\begin{aligned}
& d_{x, y, z} \circ\left(a_{m} \oplus c_{r, s, t}\right)=d_{x, y, z} \circ a_{m} \oplus d_{x, y, z} \circ c_{r, s, t}=c_{r, s, m+t-x s-y r} . \\
& a_{n} \circ\left(a_{m} \oplus d_{r, s, t}\right)=a_{n} \circ a_{m} \oplus a_{n} \circ d_{r, s, t}=a_{n}, \\
& b_{n} \circ\left(a_{m} \oplus d_{r, s, t}\right)=b_{n} \circ a_{m} \oplus b_{n} \circ d_{r, s, t}=d_{r, s, n+t-m}, \\
& c_{x, y, z} \circ\left(a_{m} \oplus d_{r, s, t}\right)=c_{x, y, z} \circ a_{m} \oplus c_{x, y, z} \circ d_{r, s, t}=c_{x, y, z-x s-y r},
\end{aligned}
$$


$d_{x, y, z} \circ\left(a_{m} \oplus d_{r, s, t}\right)=d_{x, y, z} \circ a_{m} \oplus d_{x, y, z} \circ d_{r, s, t}=d_{x+r, y+s, z+t-m-x s-y r}$.

$a_{n} \circ\left(c_{x, y, z} \oplus c_{r, s, t}\right)=a_{n} \circ c_{x, y, z} \oplus a_{n} \circ c_{r, s, t}=0$,

$b_{n} \circ\left(c_{x, y, z} \oplus c_{r, s, t}\right)=b_{n} \circ c_{x, y, z} \oplus b_{n} \circ c_{r, s, t}=c_{x+r, y+s, z+t}$,

$c_{i, k, m} \circ\left(c_{x, y, z} \oplus c_{r, s, t}\right)=c_{i, k, m} \circ c_{x, y, z} \oplus c_{i, k, m} \circ c_{r, s, t}=a_{i(y+s)+k(x+r)}($ when $i(y+s)+$ $k(x+r) \neq 0)$ or $0($ when $i(y+s)+k(x+r)=0)$,

$d_{i, k, m} \circ\left(c_{x, y, z} \oplus c_{r, s, t}\right)=d_{i, k, m} \circ c_{x, y, z} \oplus d_{i, k, m} \circ c_{r, s, t}=c_{x+r, y+s, z+t-i(y+s)-k(x+r)}$.

For $m \leq k$,

$a_{n} \circ\left(a_{m} \oplus b_{k}\right)=a_{n} \circ a_{m} \oplus a_{n} \circ b_{k}=a_{n}$,

$b_{n} \circ\left(a_{m} \oplus b_{k}\right)=b_{n} \circ a_{m} \oplus b_{n} \circ b_{k}=b_{n+k-m}$,

$c_{x, y, z} \circ\left(a_{m} \oplus b_{k}\right)=c_{x, y, z} \circ a_{m} \oplus c_{x, y, z} \circ b_{k}=c_{x, y, z}$,

$d_{x, y, z} \circ\left(a_{m} \oplus b_{k}\right)=d_{x, y, z} \circ a_{m} \oplus d_{x, y, z} \circ b_{k}=d_{x, y, z+k-m}$.

For $i \leq r$ and $k \leq s$ and $(r-i)^{2}+(s-k)^{2} \neq 0$,

$a_{n} \circ\left(c_{i, k, m} \oplus d_{r, s, t}\right)=a_{n} \circ c_{i, k, m} \oplus a_{n} \circ d_{r, s, t}=a_{n}$,

$b_{n} \circ\left(c_{i, k, m} \oplus d_{r, s, t}\right)=b_{n} \circ c_{i, k, m} \oplus b_{n} \circ d_{r, s, t}=d_{r-i, s-k, n+t-m}$,

$c_{x, y, z} \circ\left(c_{i, k, m} \oplus d_{r, s, t}\right)=c_{x, y, z} \circ c_{i, k, m} \oplus c_{x, y, z} \circ d_{r, s, t}=c_{x, y, z-x(s-k)-y(r-i)}$,

$d_{x, y, z} \circ\left(c_{i, k, m} \oplus d_{r, s, t}\right)=d_{x, y, z} \circ c_{i, k, m} \oplus d_{x, y, z} \circ d_{r, s, t}=d_{x+r-i, y+s-k, z+t-m-x(s-k)-y(r-i)}$.

For $i=r$ and $k=s$ and $m \leq t$,

$a_{n} \circ\left(c_{i, k, m} \oplus d_{r, s, t}\right)=a_{n} \circ c_{i, k, m} \oplus a_{n} \circ d_{r, s, t}=a_{n}$,

$b_{n} \circ\left(c_{i, k, m} \oplus d_{r, s, t}\right)=b_{n} \circ c_{i, k, m} \oplus b_{n} \circ d_{r, s, t}=b_{n+t-m}$,

$c_{x, y, z} \circ\left(c_{i, k, m} \oplus d_{r, s, t}\right)=c_{x, y, z} \circ c_{i, k, m} \oplus c_{x, y, z} \circ d_{r, s, t}=c_{x, y, z}$,

$d_{x, y, z} \circ\left(c_{i, k, m} \oplus d_{r, s, t}\right)=d_{x, y, z} \circ c_{i, k, m} \oplus d_{x, y, z} \circ d_{r, s, t}=d_{x, y, z+t-m}$.

We verify (SEA4), we omit the trivial cases about 0,1 :

$a_{n} \circ\left(a_{m} \circ a_{k}\right)=\left(a_{n} \circ a_{m}\right) \circ a_{k}=0$,

$a_{n} \circ\left(a_{m} \circ b_{k}\right)=b_{k} \circ\left(a_{n} \circ a_{m}\right)=a_{m} \circ\left(a_{n} \circ b_{k}\right)=0$,

$a_{n} \circ\left(a_{m} \circ c_{r, s, t}\right)=c_{r, s, t} \circ\left(a_{n} \circ a_{m}\right)=a_{m} \circ\left(a_{n} \circ c_{r, s, t}\right)=0$,

$a_{n} \circ\left(a_{m} \circ d_{r, s, t}\right)=d_{r, s, t} \circ\left(a_{n} \circ a_{m}\right)=a_{m} \circ\left(a_{n} \circ d_{r, s, t}\right)=0$,

$a_{n} \circ\left(b_{m} \circ b_{k}\right)=b_{k} \circ\left(a_{n} \circ b_{m}\right)=b_{m} \circ\left(a_{n} \circ b_{k}\right)=a_{n}$,

$a_{n} \circ\left(b_{m} \circ c_{r, s, t}\right)=c_{r, s, t} \circ\left(a_{n} \circ b_{m}\right)=b_{m} \circ\left(a_{n} \circ c_{r, s, t}\right)=0$,

$a_{n} \circ\left(b_{m} \circ d_{r, s, t}\right)=d_{r, s, t} \circ\left(a_{n} \circ b_{m}\right)=b_{m} \circ\left(a_{n} \circ d_{r, s, t}\right)=a_{n}$,

$a_{n} \circ\left(c_{i, k, m} \circ c_{r, s, t}\right)=c_{r, s, t} \circ\left(a_{n} \circ c_{i, k, m}\right)=c_{i, k, m} \circ\left(a_{n} \circ c_{r, s, t}\right)=0$, 


$$
\begin{aligned}
& a_{n} \circ\left(c_{i, k, m} \circ d_{r, s, t}\right)=d_{r, s, t} \circ\left(a_{n} \circ c_{i, k, m}\right)=c_{i, k, m} \circ\left(a_{n} \circ d_{r, s, t}\right)=0, \\
& a_{n} \circ\left(d_{i, k, m} \circ d_{r, s, t}\right)=d_{r, s, t} \circ\left(a_{n} \circ d_{i, k, m}\right)=d_{i, k, m} \circ\left(a_{n} \circ d_{r, s, t}\right)=a_{n}, \\
& b_{n} \circ\left(b_{m} \circ b_{k}\right)=b_{k} \circ\left(b_{n} \circ b_{m}\right)=b_{m+n+k}, \\
& b_{n} \circ\left(b_{m} \circ c_{r, s, t}\right)=c_{r, s, t} \circ\left(b_{n} \circ b_{m}\right)=b_{m} \circ\left(b_{n} \circ c_{r, s, t}\right)=c_{r, s, t}, \\
& b_{n} \circ\left(b_{m} \circ d_{r, s, t}\right)=d_{r, s, t} \circ\left(b_{n} \circ b_{m}\right)=b_{m} \circ\left(b_{n} \circ d_{r, s, t}\right)=d_{r, s, n+m+t}, \\
& b_{n} \circ\left(c_{i, k, m} \circ c_{r, s, t}\right)=c_{r, s, t} \circ\left(b_{n} \circ c_{i, k, m}\right)=c_{i, k, m} \circ\left(b_{n} \circ c_{r, s, t}\right)=a_{i s+k r} \text { (when is }+k r \neq
\end{aligned}
$$

0) or $0($ when is $+k r=0)$,

$$
\begin{aligned}
& b_{n} \circ\left(c_{i, k, m} \circ d_{r, s, t}\right)=d_{r, s, t} \circ\left(b_{n} \circ c_{i, k, m}\right)=c_{i, k, m} \circ\left(b_{n} \circ d_{r, s, t}\right)=c_{i, k, m-i s-k r}, \\
& b_{n} \circ\left(d_{i, k, m} \circ d_{r, s, t}\right)=d_{r, s, t} \circ\left(b_{n} \circ d_{i, k, m}\right)=d_{i, k, m} \circ\left(b_{n} \circ d_{r, s, t}\right)=d_{i+r, k+s, n+m-t-i s-k r}, \\
& c_{x, y, z} \circ\left(c_{i, k, m} \circ c_{r, s, t}\right)=c_{r, s, t} \circ\left(c_{x, y, z} \circ c_{i, k, m}\right)=0, \\
& c_{x, y, z} \circ\left(c_{i, k, m} \circ d_{r, s, t}\right)=d_{r, s, t} \circ\left(c_{x, y, z} \circ c_{i, k, m}\right)=c_{i, k, m} \circ\left(c_{x, y, z} \circ d_{r, s, t}\right)=
\end{aligned}
$$

$a_{x k+y i}($ when $x k+y i \neq 0)$ or $0($ when $x k+y i=0)$,

$$
c_{x, y, z} \circ\left(d_{i, k, m} \circ d_{r, s, t}\right)=d_{r, s, t} \circ\left(c_{x, y, z} \circ d_{i, k, m}\right)=d_{i, k, m} \circ\left(c_{x, y, z} \circ d_{r, s, t}\right)=
$$

$c_{x, y, z-x(k+s)-y(i+r)}$,

$$
d_{x, y, z} \circ\left(d_{i, k, m} \circ d_{r, s, t}\right)=d_{r, s, t} \circ\left(d_{x, y, z} \circ d_{i, k, m}\right)=d_{x+i+r, y+k+s, z+m+t-(i s+k r+x k+x s+y i+y r)} .
$$

So $\left(E_{0}, 0,1, \oplus, \circ\right)$ is a sequential effect algebra.

Our main result is:

Theorem 1. The average value inequality does not always hold in sequential effect algebras.

Proof. In fact, in $\left(E_{0}, 0,1, \oplus, \circ\right), c_{1,0,0} \perp c_{0,1,0}, c_{1,0,0} \oplus c_{0,1,0}=c_{1,1,0} \cdot c_{1,0,0} \circ c_{0,1,0}=$ $a_{1}, a_{1} \perp a_{1}, a_{1} \oplus a_{1}=a_{2}$. But $2\left(c_{1,0,0} \circ c_{0,1,0}\right)=2 a_{1}=a_{1} \oplus a_{1}=a_{2},\left(c_{1,0,0}\right)^{2}=$ $c_{1,0,0} \circ c_{1,0,0}=0,\left(c_{0,1,0}\right)^{2}=c_{0,1,0} \circ c_{0,1,0}=0$, so $2\left(c_{1,0,0} \circ c_{0,1,0}\right) \not L\left(c_{1,0,0}\right)^{2} \oplus\left(c_{0,1,0}\right)^{2}$.

Remarks. Recently, the 2 th problem, the 3 th problem, the 17 th problem and the 20th problem of Gudder have also been answered ([6-9]).

\section{References}

[1]. Foulis, D J, Bennett, M K. Effect algebras and unsharp quantum logics. Found Phys 24 (1994), 1331-1352.

[2]. Gudder, S. Sharply dominating effect algebras. Tatra Mt. Math. Publ., 15(1998), 23-30. 
[3]. Gudder, S, Nagy, G. Sequential quantum measurements. J. Math. Phys. $42(2001), 5212-5222$.

[4]. Gudder, S, Greechie, R. Sequential products on effect algebras. Rep. Math. Phys. 49(2002), 87-111.

[5]. Gudder, S. Open problems for sequential effect algebras. Inter. J. Theory. Phys. 44 (2005), 2219-2230.

[6]. Weihua Liu, Junde Wu. The Uniqueness Problem of Sequence Product on Operator Effect Algebra $\mathcal{E}(H)$. J. Physi. A (Accepted to appear).

[7]. Jun Shen, Junde Wu. Not each sequential effect algebra is sharply dominating. Physics Letter A (Accepted to appear).

[8]. Jun Shen, Junde Wu. Remarks on the sequential effect algebras. Report Math. Physi. (Accepted to appear).

[9]. Jun Shen, Junde Wu. The square root is not unique in sequential effect algebras. (To appear). 